\title{
Alterações tireoidianas \\ associadas à radiação externa em crianças e adolescentes
}

\author{
Thyroid disorders associated with external \\ radiation in children and adolescents
}

Cassiane Cardoso Bonato', Regina Helena Elnecave'

\section{SUMÁRIO}

O efeito da radiação ionizante sobre a tireoide vem sendo estudado há várias décadas, e os acidentes nucleares têm sido a maior fonte de informação. Existe associação de hipotireoidismo, hipertireoidismo, nódulos e câncer de tireoide com a radiação, mas os limiares de dose, mecanismos de lesão e alguns fatores de risco ainda não estão bem estabelecidos. Crianças são mais suscetíveis à lesão tireoidiana por radiação e necessitam de seguimento prolongado após a exposição. Esse tema adquire maior relevância atualmente, pois um grande número de pessoas tratadas com radioterapia para câncer na infância sobrevive e poderá apresentar sequelas. Exames radiodiagnósticos também representam fonte de exposição à radiação na população pediátrica. Nesta revisão, analisamos as diferentes alterações clínico-patológicas e os mecanismos de lesões tireoidianas provocadas por tratamento radioterápico e tomografia computadorizada em crianças e adolescentes. É importante conhecer esses dados para prevenção, detecção precoce e tratamento da disfunção tireoidiana. Arq Bras Endocrinol Metab. 2011;55(6):359-66

Descritores

Hipotireoidismo; criança; neoplasia; radioterapia

\section{SUMMARY}

The effects of ionizing radiation on the thyroid have been studied for several decades, and nuclear accidents are the major source of information about the subject. There is an association of hypothyroidism, hyperthyroidism, thyroid nodules and cancer with radiation, but the threshold dose, mechanism of injury, and some risk factors have not been fully established. Children are more susceptible to thyroid injury caused by radiation and require prolonged follow-up after exposure. This issue is especially relevant nowadays, since a large number of people treated with radiation for childhood cancer survive and may have sequelae. Diagnostic radiology tests also represent a source of exposure to radiation in the pediatric population. In this review, we analyze different clinical and pathological changes, and the mechanisms of thyroid lesions caused by radiotherapy and computed tomography in children and adolescents. It is important to understand these data for prevention, early detection, and treatment of thyroid dysfunction. Arq Bras Endocrinol Metab. 2011;55(6):359-66

\section{Keywords}

Hypothyroidism; child; neoplasm; radiotherapy

\section{INTRODUÇÃO}

A exposição à radiação externa ionizante apresenta diversas origens, como a exposição ambiental natural, um voo de avião, acidentes atômicos, exames diag-
1 Serviço de Endocrinologia do Hospital de Clínicas de Porto Alegre, Universidade Federal do Rio Grande do Sul (UFRGS), Porto Alegre, RS, Brasil
Correspondência para: Regina Helena Elnecave Serviço de Endocrinologia, Hospital de Clínicas de Porto Alegre Rua Ramiro Barcelos, 2350, prédio $12,4^{\circ}$ andar 90035-003 - Porto Alegre, RS, Brasil rhelnecave@gmail.com

Recebido em 24/Fev/2011 Aceito em 23/Ago/2011 nósticos e tratamentos médicos (Tabela 1). Exemplos de radiação ionizante incluem os raios $\mathrm{X}$ e gama, que não possuem massa, têm alta energia e são formas penetrantes de radiação eletromagnética. A dose de radiação 
Tabela 1. Magnitude da exposição à radiação de várias fontes*

\begin{tabular}{lc}
\hline Fonte de exposição & $\begin{array}{c}\text { Dose aproximada } \\
\text { em mSievert (mSv) }\end{array}$ \\
\hline Voo de Nova York a Londres & 0,037 \\
Raio X de tórax (póstero-anterior e lateral) & 0,14 \\
Raio X panorâmico dentário & 0,7 \\
Radiação ambiental natural de base & $3 /$ ano \\
Mamografia (dose para mama) & $2-4$ \\
Cateterismo cardíaco & $12-40$ \\
Tomografia computadorizada de tórax & $20-30$ \\
Tomografia computadorizada abdominal pediátrica & 25 \\
(dose para o estômago) & \\
Tomografia computadorizada de crânio & $30-50$ \\
Sobreviventes da bomba atômica & 200 \\
(Coorte Life-Span Study) & \\
Terapia com irradiação corporal total (TBI) & 8000
\end{tabular}

* Adaptado do Low Dose Radiation Research Program, coordenado por N. F. Metting (disponível em: http://lowdose.energy.gov/imagegallery.aspx).

absorvida é medida em Gray (Gy) (1 joule de energia para $1 \mathrm{~kg}$ de massa) - dose absorvida por um órgão ou tecido específico por unidade de massa. A lesão biológica provocada por uma unidade de radiação (dose efetiva) é expressa em Sievert (Sv) e varia conforme o tipo de radiação (alfa, beta ou gama). No caso de raios X e gama, l Gy é igual a l Sv. Os efeitos da radiação em seres humanos vêm sendo estudados há várias décadas, principalmente nos sobreviventes das bombas atômicas no Japão, quando se observou a associação entre câncer e radiação. Crianças são mais sensíveis às lesões causadas pela radiação em decorrência da maior replicação celular e por apresentarem uma expectativa de vida maior para o aparecimento de alterações.

Tem-se observado um interesse crescente em identificar danos em tecidos biológicos sadios expostos à radiação médica (diagnóstica e terapêutica). O uso de exames radiodiagnósticos tem aumentado exponencialmente, em especial o uso da tomografia computadorizada, e a dose de radiação não pode ser negligenciada (1). Na radioterapia, doses mais elevadas de radiação são dirigidas ao tumor ou tecido-alvo, mas pode haver dispersão para tecidos normais próximos (2). Essa modalidade de tratamento do câncer encontra-se frequentemente associada a sequelas precoces ou tardias e tem sido alvo de diversos estudos.

A tireoide é particularmente suscetível aos efeitos da radiação e está frequentemente envolvida no campo de irradiação diagnóstica ou terapêutica, podendo apresentar alterações funcionais e estruturais. Nesta re- visão, serão abordados os efeitos da radiação ionizante externa sobre a tireoide e suas consequências clínicas em crianças e adolescentes submetidos à tomografia computadorizada ou à radioterapia.

\section{EFEITOS BIOLÓGICOS DA RADIAÇÃO IONIZANTE}

Apesar de a radiação ionizante ser um agente terapêutico usado para diminuir ou parar o crescimento tumoral, a exposição de tecidos normais durante o tratamento pode resultar em câncer. $\mathrm{O}$ potencial carcinogênico dessa forma de radiação depende das mutações induzidas no DNA e da capacidade individual de reparo (3).

A radiação ionizante é liberada por átomos com excesso de energia, massa ou ambos. Esses átomos emitem o excedente de energia (por exemplo, raios gama) ou massa (por exemplo, partículas alfa) para tornarem-se estáveis. A radiação ionizante lesa os tecidos humanos de várias formas, e os efeitos diretos dessa radiação no microambiente tecidual são desencadeados pela deposição de energia nas macromoléculas, rompendo estruturas atômicas do tecido onde atua e produzindo modificações químicas e biológicas. Há interação direta com alvos como o RNAm, DNA e proteínas, rompendo suas ligações covalentes e quebrando sua estrutura irreversivelmente. Além disso, $60 \%$ do dano tecidual provocado por raios X e gama pode ser explicado pelos efeitos indiretos da radiação, que ocorrem devido à reação das partículas ionizadas com a água livre, produzindo espécies reativas de oxigênio e radicais livres, o que amplifica a lesão por meio da interação com lipídios, membranas e outras moléculas de oxigênio (4). Assim, as estruturas da arquitetura celular adjacente e a informação genômica são rompidas (Figura 1). A radiação ionizante causa uma ruptura celular que pode resultar na morte da célula. No entanto, a maioria dos tipos celulares não manifesta lesão até que muitas mitoses ocorram.

A lesão do DNA induz a uma resposta de estresse do ambiente intracelular, com ativação de proteínas que facilitam o reparo e previnem a proliferação de células danificadas. De forma semelhante, citocinas e fatores de crescimento atuam no parênquima e estroma para modular o comportamento e fenótipos celulares. A radiação pode, também, ativar o TGF- $\beta$ existente na matriz extracelular, que funciona como um supressor da tumorigênese e como um marcador de inflamação (5). Além disso, ocorre acúmulo de macrófagos que têm o fenótipo de fagócitos ativados, acompanhados por marginação e infiltração dos tecidos por neutrófilos, que são sinais clássicos da resposta inflamatória (6). 


\section{EFEITO DIRETO E INDIRETO DA RADIAÇÃO IONIZANTE}

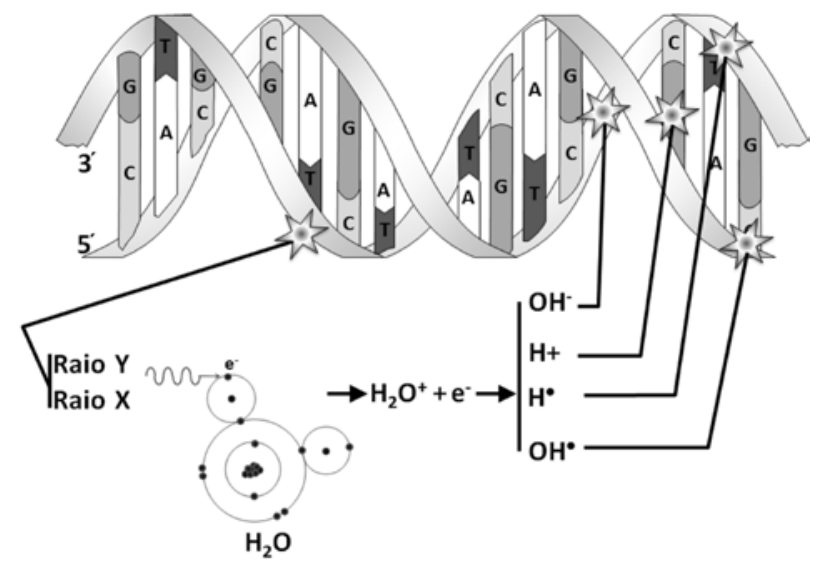

Figura 1. A lesão direta do DNA ocasiona a quebra de suas ligações estruturais. Na lesão indireta, há deslocamento de elétron (e-) da molécula de água $\left(\mathrm{H}_{2} \mathrm{O}\right)$, que se torna um íon água positivo $\left(\mathrm{H}_{2} \mathrm{O}^{+}\right)$. 0 elétron reagirá com outra molécula de água formando $\mathrm{H}_{2} \mathrm{O}^{-}$, que se dissocia em íon hidroxila $\left(\mathrm{OH}^{-}\right)$e radical livre hidrogênio $\left(\mathrm{H}^{\bullet}\right)^{2}$. 0 íon água positivo $\left(\mathrm{H}_{2} \mathrm{O}^{+}\right)$se dissocia em íon hidrogênio positivo $\left(\mathrm{H}^{+}\right)$e radical livre hidroxila $\left(\mathrm{OH}^{\bullet}\right)$. Os íons e radicais livres são altamente reativos com as estruturas celulares.

$\mathrm{Na}$ tireoide, a radiação pode inibir ou ativar funções específicas do epitélio folicular, reduzir o número de folículos funcionantes, alterar a vascularização ou a permeabilidade vascular e induzir reações imunológicas. Os achados histológicos de tireoides irradiadas variam substancialmente dependendo da dose de radiação e do intervalo que se segue à exposição. As alterações agudas, como as observadas em 3 a 6 semanas após a exposição a acidentes atômicos, incluem diminuição do tamanho dos folículos com afinamento do epitélio cuboidal dos tecidos. As modificações crônicas observadas em glândulas tireoidianas expostas durante a infância a baixas doses de irradiação externa incluem hiperplasia folicular focal, tireoidite linfocítica crônica, adenomas únicos ou múltiplos e carcinomas de tireoide papilares, foliculares ou mistos (7).

Um possível mecanismo de alteração da função tireoidiana induzida por radiação é a lesão celular direta, com exposição ao sistema imunológico, levando à produção de autoanticorpos ou à superativação de linfócitos $\mathrm{T}$ helper (8). Embora alguns estudos de sobreviventes de bombas atômicas e do acidente nuclear de Chernobyl tenham demonstrado maior prevalência de autoanticorpos tireoidianos, diretamente proporcional ao grau de exposição à radiação (9), outros não confirmaram esses resultados (10). Em pacientes submetidos à radioterapia externa, a frequência de doenças autoimunes da tireoide (tireoidite de Hashimoto, doença de Graves e oftalmopatia de Graves) é significativamente maior do que nos controles não tratados $(11,12)$.

\section{TOMOGRAFIA COMPUTADORIZADA E TIREOIDE}

Sabe-se que altas doses de radiação ionizante (> 100 $\mathrm{mSv}$ ) levam a consequências deletérias em seres humanos, incluindo, mas não exclusivamente, a indução de câncer. Com doses mais baixas, os riscos são menos claros. Grande parte da informação que dispomos vem de estudos de sobreviventes da bomba atômica no Japão, observando-se um aumento global de câncer naqueles que receberam doses entre 5 e $150 \mathrm{mSv}$ (13). No entanto, a dose de radiação resultante de exames radiológicos varia entre 3 e $30 \mathrm{mSv}$, sendo necessários estudos epidemiológicos com amostras maiores para quantificar com precisão os riscos para doses tão pequenas (14). A exposição à radiação de procedimentos médicos por imagem em geral não é monitorada e os dados de estudos longitudinais nessa área são escassos, apesar de alguns tipos de procedimentos serem realizados múltiplas vezes no mesmo paciente. A crescente utilização de exames radiológicos tem gerado preocupação com relação ao risco de lesão por órgão para diferentes doses. A tomografia computadorizada, por exemplo, utiliza vários cortes de raios $\mathrm{X}$ em múltiplos planos, sendo um dos exames que mais contribui para a dose efetiva total, conforme uma grande coorte retrospectiva norte-americana (1).

A tireoide é um órgão sensível à radiação e, com frequência, encontra-se incidentalmente exposta na área de estudos tomográficos de tórax, cabeça e pescoço. Mais de um terço de todas as tomografias computadorizadas realizadas envolvem a região da cabeça $\mathrm{e}$ pescoço (15). Um estudo de dosimetria realizado em crianças demonstrou que a tireoide pode receber doses de 15,2 a 52 mGy em tomografias da região cervical, o que poderia aumentar os casos de malignidade em mais de 390 por milhão de pessoas expostas (16). Nas tomografias de cabeça e tórax, a tireoide também pode receber doses de espalhamento de até 8,7 e $21 \mathrm{mGy}$, respectivamente $(16,17)$. Berrington de González e cols. estimaram que haveria um excesso de 1.200 casos futuros de câncer de tireoide resultantes de tomografias computadorizas realizadas nos Estados Unidos em 2007 (18). Não existem estudos, até o momento, sobre o risco de alterações funcionais e de autoimunidade da tireoide por uso de exames tomográficos.

\section{RADIOTERAPIA EXTERNA E TIREOIDE}

Aproximadamente $70 \%$ das crianças com câncer ficam curadas ou apresentam remissão prolongada com os 
tratamentos atuais (19). A radioterapia é uma modalidade de tratamento do câncer frequentemente associada a sequelas precoces ou tardias, as quais requerem diagnóstico e intervenção. A tireoide pode estar direta ou indiretamente exposta à radiação quando outros órgãos são tratados. A principal disfunção tireoidiana após a irradiação direta ou incidental da glândula é o hipotireoidismo, mas também podem ocorrer tireoidite autoimune, doença de Graves, oftalmopatia de Graves, tireoidite silenciosa, cistos e nódulos benignos ou malignos (11).

\section{HIPOTIREOIDISMO E RADIOTERAPIA}

O hipotireoidismo primário é a consequência clínica mais comum da irradiação externa para a região cervical, principalmente com altas doses (30-70 Gy) (8). Pacientes irradiados submetidos a screening bioquímico periódico frequentemente apresentam hipotireoidismo subclínico (11). A incidência de hipotireoidismo clínico e subclínico varia substancialmente $(4 \%-49 \%)$ conforme as técnicas, as doses e a frequência de radiação. Em uma população de adultos e crianças irradiadas na região cervical para tratamento de doença de Hodgkin, o hipotireoidismo clínico ou subclínico foi encontrado em $47 \%$ dos pacientes 27 anos após o tratamento. Aproximadamente metade desses casos se manifestou até cinco anos após a radioterapia (11). Dados do maior estudo de sobreviventes de câncer na infância, o Childhood Cancer Survivor Study (CCSS) (20), mostram que a incidência de hipotireoidismo em pacientes que tiveram doença de Hodgkin foi significativamente maior nos irradiados do que no grupo controle (irmãos hígidos) $(\mathrm{RR}=17,1 ; \mathrm{p}<0,0001)$ e a dose de radiação para a tireoide foi o fator de risco mais importante, sendo que o risco relativo aumentou em 1,06 por Gray $(\mathrm{p}<0,0001)$. Aproximadamente metade dos pacientes manifestaram hipotireoidismo em 2 a 7 anos após a terapia, com um declínio da incidência após esse período. O desenvolvimento de hipotireoidismo é diretamente proporcional à dose de radiação que atinge a tireoide, principalmente quando maior que 20 Gy $(11,20)$.

Outros fatores associados ao maior risco de hipotireoidismo após irradiação cervical, como a idade do indivíduo no momento do tratamento radioterápico, o sexo e o uso associado de quimioterápicos, foram estudados. Embora a sensibilidade da tireoide à radiação pareça ser elevada em crianças pequenas, a incidência de hipotireoidismo mostrou-se maior nos pacientes trata- dos dos 15 aos 20 anos (39\%) do que naqueles tratados antes dos 5 anos de idade (15\%) (11). Um possível fator de confusão poderia ter sido o uso de doses menores de radiação nos mais jovens. No entanto, esse achado foi confirmado, demonstrando a idade maior de 15 anos como um fator de risco independente (20). Em adultos, o sexo feminino foi identificado como um fator de risco (11), achado controverso em crianças e adolescentes (20). Os efeitos tardios do tratamento do câncer na infância são descritos predominantemente em relação à radioterapia. Na maioria dos estudos, a quimioterapia não tem demonstrado efeito adicional ou isolado sobre a função ou o aparecimento de nódulos de tireoide em indivíduos tratados para câncer na infância (21). No entanto, já foi observado efeito deletério adicional sobre a função tireoidiana em pacientes tratados com radioterapia para tumores cerebrais $(22)$.

O hipotireoidismo também foi documentado após exposição incidental da tireoide à radiação, ou seja, quando a glândula está fora do campo de irradiação. Crianças tratadas para tumores do sistema nervoso central (SNC) apresentaram hipotireoidismo em 20\% a $68 \%$ dos casos (22). Em irradiação corporal total para o transplante de medula óssea a incidência de hipotireoidismo clínico ou subclínico foi de $25 \%$ a $73 \%(12,23)$, sendo menor quando fracionada do que após dose única para regimes preparatórios (12).

Em um estudo retrospectivo, hipotireoidismo bioquímico foi observado em 23/59 (39\%) pacientes tratados com radiação externa para diferentes áreas corporais durante a infância (média de idade 7,6 $\pm 3,4$ anos). Essa foi uma alteração precoce, em média $3,5 \pm 1,9$ anos após a radioterapia. $\mathrm{O}$ fator mais fortemente associado ao hipotireoidismo foi a proximidade da zona irradiada (área de risco) com o leito tireoidiano. Todos os pacientes apresentaram anticorpos antitireoperoxidase normais. $\mathrm{O}$ volume tireoidiano nos pacientes com hipotireoidismo foi significativamente menor do que naqueles com função tireoidiana normal $(\mathrm{p}<0,001)(24)$.

Anticorpos antitireoidianos foram mais frequentemente encontrados em pacientes que receberam radiação externa na região cervical do que em pacientes não tratados (12), achado não confirmado por outros estudos, que atribuíram o hipotireoidismo à redução do número de células foliculares $(24,25)$.

O hipotireoidismo secundário também pode ocorrer após irradiação da região hipotalâmico-hipofisária, de tumores cerebrais ou de alguns cânceres de cabeça e pescoço $(22,26,27)$. O intervalo médio de tempo para a 
detecção de hipotireoidismo central é maior do que para o hipotireoidismo primário, chegando até 19 anos. Esse fato pode indicar que as avaliações feitas a curto prazo subestimem a incidência do problema. $\mathrm{O}$ risco de hipotireoidismo central está diretamente relacionado à dose de irradiação (22). Os outros eixos hipotalâmico-hipofisários são mais vulneráveis à radiação do que o eixo hipotálamo-hipófise-tireoide, afetado em 3\% a 6\% dos irradiados para tumores cerebrais não hipofisários $\mathrm{e}, \mathrm{em}$ geral, associado a doses maiores do que 50 Gy $(26,28)$.

$\mathrm{O}$ bloqueio da atividade metabólica e proliferativa da tireoide por meio do uso transitório de levotiroxina durante a radioterapia em crianças mostrou-se protetor contra a lesão funcional (hipotireoidismo primário) (29), mas deve ser comprovado por outros ensaios clínicos.

\section{HIPERTIREOIDISMO E RADIOTERAPIA}

Diversos casos de tireoidite tireotóxica após irradiação externa, incluindo o leito tireoidiano, foram relatados de duas semanas a vários anos após o tratamento $(30,31)$. O hipertireoidismo parece ser uma complicação precoce após a irradiação externa, mas permanece pouco diagnosticado. Não se sabe da história natural dessa disfunção, pois não se dispõem de estudos prospectivos maiores. Como o número de casos relatados na literatura é pequeno, é difícil determinar a verdadeira incidência do hipertireoidismo e estabelecer quais seriam os fatores de risco mais importantes. Até o momento, a variável associada ao maior risco foi a maior dose de radiação $(11,20)$.

Em geral, o quadro clínico de hipertireoidismo é idêntico ao da doença de Graves e caracterizado pelo aumento da glândula tireoide, níveis elevados de hormônios tireoidianos, níveis suprimidos de TSH, aumento da captação tireoidiana de iodo e aumento de autoanticorpos para a tireoide. O risco de doença de Graves é 7 a 20 vezes maior em pacientes irradiados em região cervical para o tratamento de doença de Hodgkin do que na população geral (11). O desenvolvimento de autoanticorpos tireoidianos pode resultar da secreção de antígenos expostos após a lesão da tireoide induzida pela radiação. Apesar de ser menos frequente que o hipotireoidismo, a doença de Graves foi descrita em dois estudos maiores, que mostraram hipertireoidismo em média 5,3 a 8 anos após o tratamento radioterápico $(11,20)$. Dos 30 pacientes com hipertireoidismo avaliados, 17 desenvolveram oftalmopatia infiltrativa. A dose de irradiação cervical e o tempo desde o tratamento da doença de Hodgkin foram preditores independentes de hipertireoidismo (20).

\section{NEOPLASIAS DE TIREOIDE E RADIOTERAPIA}

Nódulos benignos e malignos são encontrados com maior frequência em pacientes expostos à radiação ionizante terapêutica ou acidental. Nódulos benignos foram as lesões nodulares mais prevalentes nos grupos expostos a acidentes nucleares, sendo mais uninodulares no Japão (32) e multinodulares em Chernobyl (33). Há correlação direta entre a incidência de adenomas tireoidianos e a dose de radiação (34). Os pacientes submetidos à irradiação terapêutica de cabeça e pescoço podem apresentar nódulos adenomatosos (30\%-90\%), bócio nodular (33\%-77\%) e cistos coloides (6\%-11\%) (35).

A associação entre radiação e carcinoma de tireoide foi inicialmente descrita por Duffy e Fitzgerald em 1950 (36). Foi observado que grande parte das crianças com carcinoma de tireoide tinha história de radioterapia. Vários estudos confirmaram essa associação (37). Em uma metanálise realizada em 1995, incluindo sete estudos, demonstrou-se, pela primeira vez, que a exposição a doses baixas ou moderadas de raio $\mathrm{X}$ ou radiação gama está associada a um risco crescente de câncer de tireoide, proporcional à dose (38). Resultados comparáveis foram descritos em um estudo caso-controle (CCSS), sendo observado, também, que esse risco diminui com a exposição a doses maiores que $30 \mathrm{~Gy}$, corroborando a hipótese de morte celular com altas doses de radiação proposta por Louis Gray em 1964 (39).

$\mathrm{O}$ risco de câncer de tireoide é maior em crianças irradiadas antes dos 5 anos de idade (40). Há correlação negativa entre a idade do paciente no momento da irradiação e o risco de segundo câncer em tireoide (38). Em crianças mais jovens, o risco maior de câncer de tireoide após irradiação craniana é atribuído à menor distância craniofacial e à rápida proliferação das células da tireoide (27). A incidência de câncer de tireoide é maior 10 a 15 anos após a exposição à radiação externa, com um tempo de latência de aproximadamente cinco anos $(37,41,42)$. O tempo de latência foi mais curto em vítimas do acidente nuclear de Chernobyl, em que o radioiodo foi um dos componentes (42).

Em um estudo recente, envolvendo 17.980 indivíduos sobreviventes de câncer na infância, o risco relativo para o aparecimento de segundo câncer em tireoide foi de 4,6 (intervalo de confiança de 95\%: 1,4-15,1; 
$\mathrm{p}=0,003)$, quando comparados aos sobreviventes não tratados com radioterapia (43). A taxa padronizada de incidência por período de seguimento revelou ser maior de 0 a 9 anos em relação a períodos posteriores. Oitenta e oito por cento dessas lesões apareceram quando a tireoide estava próxima ou envolvida no campo de irradiação, sendo o risco maior nos pacientes que fizeram tratamento de linfomas Hodgkin e não Hodgkin, quando comparados aos tratados para leucemia e tumores do SNC. A taxa de câncer de tireoide observada sobre a esperada foi maior no sexo masculino do que no feminino após a radiação externa, embora essa relação seja o inverso na população geral, consistente com dados do CCSS (44).

O carcinoma papilar é a variante histológica mais comum após a irradiação, representando $78 \%$ dos casos de segundo câncer em tireoide nos pacientes tratados para outro câncer (41) e 93,8\% em vítimas do acidente nuclear de Chernobyl (45). A prevalência de rearranjos RET/PTC é maior em carcinomas papilares de pacientes com história de exposição à radiação incidental (67\%-87\%) ou à irradiação terapêutica externa (52\%$84 \%)$ do que em pacientes não irradiados $(46,47)$. Nas crianças afetadas pelo acidente nuclear de Chernobyl, os tumores de aparecimento mais precoce foram associados à mutação RET/PTC3, enquanto aqueles com maior período de latência foram associados à RET/ PTCl (47). São conflitantes os dados quanto à agressividade dos tumores de tireoide após radiação externa $(37,47,48)$.

\section{AVALIAÇÃO E SEGUIMENTO PÓS-RADIOTERAPIA}

Tratando-se de crianças, o crescimento e o desenvolvimento neuropsicológico são fatores importantes. Portanto, recomenda-se que os pacientes que receberam radiação direta ou incidental na região tireoidiana ou hipotalâmico-hipofisária sejam seguidos e avaliados pelo menos anualmente para história de sintomas de disfunção tireoidiana, exame clínico e medida de T4 e TSH (49). Segundo o guideline escocês "Long term follow up of survivors of childhood cancer", a função tireoidiana deve ser aferida logo após o final da radioterapia e repetida pelo menos anualmente $(50)$.

O diagnóstico de hipotireoidismo central é facilmente realizado em pacientes com nível baixo de $\mathrm{T} 4$ com concentração normal-baixa de TSH. Em alguns casos, no entanto, o TSH pode estar elevado. O teste do TRH pode não ser útil nesses casos, pois grande par- te dos pacientes com hipotireoidismo central apresenta teste do TRH normal ou semelhante aos pacientes com hipotireoidismo primário (51). Assim, a única forma de diagnosticar essa condição seria por meio do decréscimo seriado dos níveis de T4 (52).

A ecografia pode revelar volume tireoidiano reduzido e alterações estruturais. Nódulos tireoidianos podem ser detectados em mais de $40 \%$ dos pacientes irradiados (53), sendo que um terço é maligno (54). O Children's Oncology Group recomenda palpação anual da tireoide, seguida por ultrassom e outros testes em caso de nódulo palpável. Em razão do alto risco de malignidade, alguns autores recomendam ultrassom periódico no primeiro ano e, posteriormente, a cada 2-3 anos $(55,56)$. Embora a ecografia aumente a detecção de câncer de tireoide, também poderá induzir a cirurgias de alguns cânceres que nunca progrediriam e de alguns nódulos benignos que não seriam adequadamente avaliados por punção por agulha fina (PAAF) (57). Deve-se realizar a punção em nódulos tireoidianos palpáveis, embora a avaliação citológica possa ser difícil pela presença de atipias celulares induzidas pela radiação. Hatipoglu e cols. demonstraram que a sensibilidade e especificidade da PAAF em pacientes irradiados são similares às encontradas na população geral (58). A Associação Americana de Tireoide recomenda que a PAAF seja realizada em nódulos menores do que o usual (menores que $1 \mathrm{~cm}$ no maior diâmetro), se houver história de exposição à radiação (59).

\section{CONCLUSÃO}

$\mathrm{Na}$ infância, a exposição da tireoide à radiação externa ionizante, mesmo com baixas doses, deve ser vista com preocupação. A tomografia computadorizada é um dos exames radiodiagnósticos que fornecem maior dose de exposição à radiação. A utilização crescente dessa técnica aplicada em larga escala, e por vezes repetidamente, torna-se um problema de saúde pública pelo risco potencial de câncer de tireoide. O uso responsável desse exame deve ser encorajado.

A lesão da tireoide também pode acontecer após o tratamento de neoplasias por radioterapia cervical ou em áreas próximas. Os sintomas de disfunção tireoidiana podem ser confundidos ou interpretados de forma errônea ao serem atribuídos à doença de base do paciente ou às medicações usadas para o tratamento da neoplasia, resultando em diagnóstico tardio da lesão glandular. Considerando-se o sucesso no tratamento do 
câncer da infância, os pacientes submetidos à radioterapia devem ter um seguimento adequado, possibilitando o diagnóstico correto e o tratamento precoce das eventuais sequelas sobre a tireoide.

Declaração: os autores declaram não haver conflitos de interesse científico neste estudo.

\section{REFERÊNCIAS}

1. Fazel R, Krumholz HM, Wang Y, Ross JS, Chen J, Ting HH, et al. Exposure to low-dose ionizing radiation from medical imaging procedures. N Engl J Med. 2009;361(9):849-57.

2. Ricardi U, Rossi G, Tessa M, Barisone E, Besenzon L, Corrias A, et al. Dosimétrie in vivo pour l'evaluation de la dose reçue par la thyroïde lors de l'irradiation cérébrale prophylactique délivrée dans le cadre du traitement des leucémies de l'enfant. Cancer Radiother. 1997;1(1):85-7.

3. Baverstock K. Radiation-induced genomic instability: a paradigm-breaking phenomenon and its relevance to environmentally induced cancer. Mutat Res. 2000;454(1-2):89-109.

4. Barcellos-Hoff MH, Brooks AL. Extracellular signaling through the microenvironment: a hypothesis relating carcinogenesis, bystander effects, and genomic instability. Radiat Res. 2001;156(5 Pt 2):618-27.

5. Bhowmick NA, Chytil A, Plieth D, Gorska AE, Dumont N, Shappell $\mathrm{S}$, et al. TGF- $\beta$ signaling in fibroblasts modulates the oncogenic potential of adjacent epithelia. Science. 2004;303(5659):848-51.

6. Lorimore SA, Coates PJ, Scobie GE, Milne G, Wright EG. Inflammatory-type responses after exposure to ionizing radiation in vivo: a mechanism for radiation-induced bystander effects? Oncogene. 2001;20(48):7085-95.

7. Fajardo LF. Pathology of radiation injury. New York: Masson Pub; 1982.

8. Jereczek-Fossa BA, Alterio D, Jassem J, Gibelli B, Tradati N, Orecchia R. Radiotherapy-induced thyroid disorders. CancerTreat Rev. 2004;30(4):369-84.

9. Pacini F, Vorontsova T, Molinaro E, Kuchinskaya E, Agate L, Shavrova $E$, et al. Prevalence of thyroid autoantibodies in children and adolescents from Belarus exposed to the Chernobyl radioactive fallout. Lancet. 1998;352(9130):763-6.

10. Fujiwara S, Carter RL, Akiyama M, Akahoshi M, Kodama K, Shimaoka K, et al. Autoantibodies and immunoglobulins among atomic bomb survivors. Radiat Res. 1994;137(1):89-95.

11. Hancock SL, Cox RS, McDougall IR. Thyroid diseases after treatment of Hodgkin's disease. N Eng J Med. 1991;325(9):599-605.

12. Tamura K, Shimaoka K, Friedman M. Thyroid abnormalities associated with treatment of malignant lymphoma. Cancer. 1981;47(11):2704-11.

13. Preston DL, Shimizu Y, Pierce DA, Suyama A, Mabuchi K. Studies of mortality of atomic bomb survivors. Report 13: solid cancer and non cancer disease mortality: 1950-1997. Radiat Res. 2003;160(4):381-407.

14. Brenner DJ, Doll R, Goodhead DT, Hall EJ, Land CE, Little JB, et al. Cancer risks attributable to low doses of ionizing radiation: assessing what we really Know. Proc Natl Acad Sci USA. 2003;100(24):13761-6.

15. Mettler FA Jr, Wiest PW, Locken JA, Kelsey CA. CT scanning: patterns of use and dose. J Radiol Prot. 2000;20(4):353-9.

16. Mazonakis M, Tzedakis A, Damilakis J, Gourtsoyiannis N. Thyroid dose from common head and neck CT examinations in children: is there an excess risk for thyroid cancer induction? Eur Radiol. 2007;17(5):1352-7.

17. Brisse HJ, Robilliard M, Savignoni A, Pierrat N, Gaboriaud G, De RyckeY, et al. Assessment of organ absorbed doses and estimation of effective doses from pediatric anthropomorphic phantom measurements for multi-detector row CT with and without automatic exposure control. Health Phys. 2009;97(4):303-14.

18. Berrington de González A, Mahesh M, Kim KP, Bhargavan M, Lewis $R$, Mettler $F$, et al. Projected cancer risks from computed tomographic scans performed in the United States in 2007. Arch Intern Med. 2009;169(22):2071-7.

19. Brasil, Instituto Nacional do Câncer (Inca) [Internet]. Particularidades do câncer infantil. Brasília: INCA [citado 2011 jan 23]. Disponível em: http://www.inca.gov.br/conteudo_view.asp?id=343.

20. Sklar C, Whitton J, Mertens A, Stovall M, Green D, Marina N, et al. Abnormalities of the thyroid in survivors of Hodgkin's disease: data from the Childhood Cancer Survivor Study. J Clin Endocrinol Metab. 2000;85(9):3227-32.

21. van Santen HM, Vulsma T, Dijkgraaf MG, Blumer RME, Heinen R, Jaspers MW, et al. No damaging effect of chemotherapy in addition to radiotherapy on the thyroid axis in young adult survivors of childhood cancer. J Clin Endocrinol Metab. 2003;88(8):3657-63.

22. Ogilvy-Stuart AL, Shalet SM, Gattamaneni HR. Thyroid function after treatment of brain tumors in children. J Pediatr. 1991;119(5):733-7.

23. Sklar CA, Kim TH, Ramsay NK. Thyroid dysfunction among long-term survivors of bone marrow transplantation. Am J Med. 1982;73(5):688-94.

24. Bonato C, Severino RF, Elnecave RH. Reduced thyroid volume and hypothyroidism in survivors of childhood cancer treated with radiotherapy. J Pediatr Endocrinol Metab. 2008;21(10):943-9.

25. van Santen HM, Van Dijk JE, Rodermond H, Vansenne F, Meertens $\mathrm{N}$, Haveman J, et al. The effect of cervical X-irradiation on activity index of thyrocytes and plasma TSH: a pre-clinical model for radiation-induced thyroid damage. J Endocrinol Invest. 2005;28(3):261-9.

26. Constine LS, Woolf PD, Cann D, Mick G, McCormick K, Raubertas $\mathrm{RF}$, et al. Hypothalamic-pituitary dysfunction after radiation for brain tumors. N Engl J Med. 1993;328(2):87-94.

27. Bessho F, Ohta K, Akanuma A, Sakata K. Dosimetry of radiation scattered to thyroid gland from prophylactic cranial irradiation for childhood leukemia. Pediatr Hematol Oncol. 1994;11(1):47-53.

28. Lam KS, Tse VK, Wang C, Yeung RT, Ho JH. Effects of cranial irradiation on hypothalamic-pituitary function--a 5-year longitudinal study in patients with nasopharyngeal carcinoma. $\mathrm{Q} \mathrm{J}$ Med. 1991;78(2086):165-76.

29. Massimino M, Gandola L, Collini P, Seregni E, Marchianò A, Serra A, et al.Thyroid-stimulating hormone suppression for protection against hypothyroidism due to craniospinal irradiation for childhood medulloblastoma/primitive neuroectodermal tumor. Int $\mathrm{J}$ Radiat Oncol Biol Phys. 2007;69(2):404-10.

30. Blitzer JB, Paolozzi FP, Gottlieb AJ, Zamkoff KW, Chung CT.Thyrotoxic thyroiditis after radiotherapy for Hodgkin's disease. Arch Intern Med. 1985;145(9):1734-35.

31. Petersen M, Keeling CA, McDougall IR. Hyperthyroidism with low radioiodine uptake after head and neck irradiation for Hodgkin's disease. J Nucl Med. 1989;30(2):255-7.

32. Morimoto I, Yoshimoto Y, Sato K, Hamilton HB, Kawamoto S, Izumi $M$, et al. Serum TSH, thyroglobulin, and thyroidal disorders in atomic bomb survivors exposed in youth: 30-year follow-up study. J Nucl Med. 1987;28(7):1115-22.

33. Nikiforov Y, Heffess CS, Korzenko AV, Fagin JA, Gnepp DR. Characteristics of follicular tumors and nonneoplastic thyroid lesions in children and adolescents exposed to radiation as a result of the Chernobyl disaster. Cancer. 1995;76(5):900-9. 
34. Ezaki H, Takeichi N, Yoshimoto Y. Thyroid cancer: epidemiological study of thyroid cancer in A-bomb survivors from extended life span study cohort in Hiroshima. J Radiat Res. 1991;32 Suppl:193-200.

35. Fjälling M,Tisell LE, Carlsson S, Hansson G, Lundberg LM, Odén A. Benign and malignant nodules after neck irradiation. Cancer. 1986;58(6):1219-24.

36. Duffy BJ Jr, Fitzgerald PJ. Cancer of the thyroid in children: a report of 28 cases. J Clin Endocrinol Metab. 1950;10(10):1296-308.

37. Archarya S, Sarafoglou K, LaQuaglia M, Lindsley S, Gerald W, Wollner $\mathrm{N}$, et al. Thyroid neoplasms after therapeutic radiation malignancies during childhood or adolescence. Cancer. 2003;97(10):2397-403.

38. Ron E, Lubin JH, Shore RE, Mabuchi K, Modan B, Pottern LM, et al. Thyroid cancer after exposure to external radiation: a pooled analysis of seven studies. Radiat Res. 1995;141(3):259-77.

39. Gray LH. Radiation biology and cancer. In: Cellular Radiation Biology, Proc Ann Symp Fundamental Cancer Research. Baltimore: Williams \& Wilkins; 1964. p. 7-25.

40. Inskip PD. Thyroid cancer after radiotherapy for childhood cancer. Med Ped Oncol. 2001;36(5):568-73.

41. Sigurdson AJ, Ronckers CM, Mertens AC, Stovall M, Smith SA, Liu $Y$, et al. Primary thyroid cancer after a first tumour in childhood (the Childhood Cancer Survivor Study): a nested case-control study. Lancet. 2005;365(9476):2014-23.

42. Massimino M, Gandola L, Mattavelli F, Pizzi N, Seregni E, Pallotti F, et al. Radiation-induced thyroid changes: a retrospective and a prospective view. Eur J Cancer. 2009;45(14):2546-51.

43. Taylor AJ, Croft AP, Palace AM, Winter DL, Reulen RC, Stiller CA, et al. Risk of thyroid cancer in survivors of childhood cancer: results from the British Childhood Cancer Survivor Study. Int J Cancer. 2009;125(10):2400-5.

44. Neglia JP, Friedman DL, YasuiY, Mertens AC, Hammond S, Stovall $M$, et al. Second malignant neoplasms in five-year survivors of childhood cancer: childhood cancer survivor study. J Natl Cancer Inst. 2001;93(8):618-29.

45. Pacini F, VorontsovaT, Demidchik EP, Molinaro E, Agate L, Romei C, et al. Post-Chernobyl thyroid carcinoma Belarus children and adolescents: comparison with naturally occurring thyroid carcinoma in Italy and France. J Clin Endocrinol Metabol. 1997;82(11):3563-9.

46. Elisei R, Romei C, Vorontsova T, Cosci B, Veremeychik V, Kuchinskaya $E$, et al. RET/PTC rearrangements in thyroid nodules: studies in irradiated and not irradiated, malignant and benign thyroid lesions in children and adults. J Clin Endocrinol Metab. 2001;86(7):3211-6.
47. Rabes HM, Demidchik EP, Sidorow JD, Lengfelder E, Beimfohr C, Hoelzel D, et al. Pattern of radiation-induced RET and NTRK1 rearrangements in 191 post-Chernobyl papillary thyroid carcinomas: biological, phenotypic, and clinical implications. Clin Cancer Res. 2000;6(3):1093-103.

48. Rubino C, Cailleux AF, Abbas M, Diallo I, Shamsaldin A, Caillou B, et al. Characteristics of follicular cell-derived thyroid carcinomas occurring after external radiation exposure: results of a case control study nested in a cohort. Thyroid. 2002;12(4):299-304.

49. Hancock SL, McDougall IR, Constine LS. Thyroid abnormalities after therapeutic external radiation. Int J Radiat Oncol Biol Phys. 1995;31(5):1165-70.

50. Scottish Intercollegiate Guidelines Network. Long term follow up of survivors of childhood cancer. A national clinical guideline [Internet]. Jan 2004 [Atualizado mar 2005]. Disponível em: http:// www.sign.ac.uk/pdf/sign76.pdf.

51. Rose SR, Lustig RH, Pitukcheewanont P, Broome DC, Burghen GA, $\mathrm{Li} \mathrm{H}$, et al. Diagnosis of hidden central hypothyroidism in survivors of childhood cancer. J Clin Endocrinol Metab. 1999;84(12):4472-9.

52. Darzy KH. Radiation-induced hypopituitarism after cancer therapy: who, how and when to test. Nat Clin Pract Endocrinol Metab. 2009;5(2):88-99.

53. Schneider AB, Recant W, Pinsky SM, Ryo UY, Bekerman C, Shore-Freedman E. Radiation-induced thyroid carcinoma. Clinical course and results of therapy in 296 patients. Ann Intern Med. 1986;105(3):405-12.

54. Roman SA. Endocrine tumors: evaluation of the thyroid nodule. Curr Opin Oncol. 2003;15(1):66-70.

55. Crom DB, Kaste SC, Tubergen DG, Greenwald CA, Sharp GB, Hudson MM. Ultrasonography for thyroid screening after head and neck irradiation in childhood cancer survivors. Med Pediatr Oncol. 1997;28(1):15-21.

56. Mihailescu DV, Collins BJ, Wilbur A, Malkin J, Schneider AB. UItrasound-detected thyroid nodules in radiation-exposed patients: changes over time. Thyroid. 2005;15(2):127-33.

57. Eden K, Mahon S, Helfand M. Screening high-risk populations for thyroid cancer. Med Pediatr Oncol. 2001;36(5):583-91.

58. Hatipoglu BA, Gierlowski T, Shore-Freedman E, Recant W, Schneider $A B$. Fine-needle aspiration of thyroid nodules in radiation-exposed patients. Thyroid. 2000;10(1):63-9.

59. Cooper DS, Doherty GM, Haugen BR, Kloos RT, Lee SL, Mandel SJ, et al. Management guidelines for patients with thyroid nodules and differentiated thyroid cancer. Thyroid. 2006;16(2):109-42. 